\title{
émulations
}

\section{Rommel Mendès-Leite - Des mots, des pratiques et des risques. Études sur le genre, les sexualités et le sida}

Maialen Pagiusco

Émulations - Revue de sciences sociales 2019. Comptes rendus critiques. En ligne

\section{Article disponible à l'adresse suivante}

https://ojs.uclouvain.be/index.php/emulations/article/view/7543

\section{Pour citer cet article}

Maialen Pagiusco, «Rommel Mendès-Leite - Des mots, des pratiques et des risques. Études sur le genre, les sexualités et le sida », Émulations, en ligne. Mise en ligne le 7 janvier 2019. DOI : 10.14428/emulations.cr.053

Distribution électronique : Université catholique de Louvain (Belgique) : ojs.uclouvain.be

(C) Cet article est mis à disposition selon les termes de la Licence Creative Commons Attribution, Pas d'Utilisation Commerciale 4.0 International. http://creativecommons.org/licenses/by-nc/4.0/

Éditeur : Émulations - Revue de sciences sociales / Presses universitaires de Louvain https://ojs.uclouvain.be/index.php/emulations

ISSN électronique : $1784-5734$

UCL PRESSES 


\title{
Rommel Mendès-Leite - Des mots, des pratiques et des risques. Études sur le genre, les sexualités et le sida
}

\begin{abstract}
Maialen Pagiusco ${ }^{1}$
Recensé : Rommel Mendès-Leite (2016), Des mots, des pratiques et des risques. Études sur le genre, les sexualités et le sida, Lyon, Presses universitaires de Lyon (" Sexualités »), 308 p.

À sa mort le 22 janvier 2016, l'anthropologue Rommel Mendès-Leite ${ }^{2}$ est unanimement salué par la communauté scientifique comme étant l'un des pionniers, en France, des études des sexualités en sciences sociales. Comme le rappelle Maks Banens, un de ses collègues à l'Université Lumière-Lyon-2, dans la préface de cet ouvrage posthume, son combat fut long, et ses travaux cantonnés durant des années dans la littérature grise ainsi que dans la collection " Sciences sociales et sida » de l'ANRS. Précisément Mendès-Leite compte parmi ceux qui ont créé en France un espace de recherche et de publication sur ces thématiques. C'est pourquoi la collection Sexualités des Presses universitaires de Lyon lui rend hommage avec la parution d'une compilation de ses principales publications, entre 1995 et 2015, portant sur les (homo)sexualités, le genre et le sida.

Les vingt années de son activité de chercheur, ainsi restituées, permettent de mesurer l'évolution de certaines notions ou approches qu'il a développées, mais surtout l'actualité des problématiques qu'il soulève. Les neuf publications sont regroupées en deux sections : "Culture des genres et des sexualités : ici et ailleurs " et "VIH et SIDA ». L'ensemble des textes permet ainsi d'interroger la construction du genre et des sexualités. L'auteur s'inscrit en effet dans la lignée des travaux relevant du constructionnisme social (Foucault, 1976 ; Weeks, 1977) qui " insiste sur [le] caractère historiquement, culturellement ou socialement situé et construit » de l'homosexualité (Broqua, 2011 : 3). Cette approche s'accompagne par ailleurs d'une démarche empirique et ethnographique faisant une place majeure au terrain (observations, entretiens menés à la fois au Brésil, dont Mendès-Leite est originaire, et en France).

$S^{\prime}$ il n'est guère possible d'entrer dans le détail de chacune des publications, trois grandes lignes directrices peuvent être dégagées suivant les termes-clés « des mots, des pratiques et des risques ", mis en avant dans un article publié il y a vingt ans avec Catherine Deschamps (Mendès-Leite, Deschamps, 1997) et qui servent de titre éponyme pour cette réédition.
\end{abstract}

\footnotetext{
${ }^{1}$ Master 2 de recherche à I'IEP de Toulouse.

${ }^{2}$ Bien qu'anthropologue, Mendès-Leite était rattaché à un laboratoire de sociologie, le Centre Max Weber, et enseignait à l'Institut de Psychologie de Lyon 2. Ces multiples affiliations soulignent bien l'intérêt de la pluridisciplinarité dans les études des sexualités.
} 
Les recherches de Mendès-Leite permettent en effet de déconstruire les « mots » et les catégories, de restituer le sens des « pratiques » et, enfin, d'interroger leurs implications sociales et les « risques » qu'elles peuvent induire.

\section{Des mots : analyser et déconstruire les catégories}

L'ouvrage débute par un article intitulé « Genre et orientations sexuelles : une question d'apparence ? » qui est tiré des premières recherches de l'auteur. II porte sur l'analyse des catégories du discours populaire pour désigner l'homosexualité masculine dans le Brésil des années 1980. Dans le langage courant, le mot « bicha » renvoie aux individus de sexe masculin qui adoptent un comportement " passif » lors de la pénétration anale et dont le "langage corporel [peut être] socialement perçu comme efféminé » (p. 35). La figure du " bicha » est associée, dans l'imaginaire des sexualités, au genre féminin. Elle s'oppose à celle du " bofe », actif lors du coït anal et donc considéré comme un hétérosexuel de genre masculin. Avec l'évolution du système de représentations machistes vers une plus grande égalité entre les genres, on voit cependant apparaître une nouvelle figure dans l' « univers homosexuel " : I' « entendido » dont le comportement sexuel est perçu comme plus mouvant (" passif » et " actif ») et qui échappe en partie au système hiérarchique fondé sur un « jeu des apparences ». L'auteur met en évidence l'imbrication entre construction des genres et construction des sexualités ainsi que l'évolution concomitante de ces deux systèmes de représentation. Si Mendès-Leite étudie ici les mutations des catégories sociales dans le temps, il s'attache, dans d'autres articles, à interroger leur variation dans l'espace.

C'est le cas d'un chapitre d'ouvrage collectif de 2012, intitulé « Inconstance des sexes et des genres dans les sociétés non occidentales », dans lequel Mendès-Leite interroge la catégorie occidentale et contemporaine $d^{\prime}$ " intersexualité ». II propose une synthèse de données ethnographiques portant sur l'articulation du système de sexe et de genre dans différentes cultures. Si parler d' ' intersexualité » relève d'une forme d'ethnocentrisme, il existe bien, dans des sociétés non-occidentales, des statuts et rôles sociaux pour les personnes qui n'entrent pas dans les classements binaires et dichotomiques de sexe/genre ${ }^{3}$. Ce sont par exemple les " Mahus » en Polynésie, les " Hijras » de l'Inde, les "Sipiniit » inuits, même si ces catégories ne recoupent pas exactement les mêmes réalités sociales.

Pour déconstruire les mots et les réalités qu'ils désignent, Mendès-Leite recourt également à l'analyse lexicale. Dans le deuxième article de l'ouvrage intitulé « L'homme, son miroir et les femmes ", il utilise cette méthode pour saisir les représentations du rôle " masculin ", à partir de questionnaires administrés à quelques cent soixante-dix étudiant·e·s de l’Université de Fortaleza, dans la région brésilienne du Nordeste. L'analyse lexicale permet ainsi d'étudier les

\footnotetext{
${ }^{3}$ On note, pour ce qui est de l'Occident, une progressive reconnaissance juridique et sociale, comme en témoigne la décision du tribunal constitutionnel de Karlsruhe qui demande au législateur allemand de supprimer la mention du sexe à l'état civil ou de reconnaître positivement les personnes non binaires en ajoutant une troisième catégorie sur les registres de naissance et les documents officiels.
} 
associations de mots et leur récurrence dans les réponses à la question « Pour vous, qu'est-ce qu'être un homme ? ". Les discours varient selon que les enquêté.e.s parlent d'un point de vue macrosocial (les « hommes " en général) ou qu'ils ou elles se réfèrent à leur propre expérience ("l'homme » qu'ils sont ou connaissent). Plusieurs représentations de la masculinité s'affrontent alors : "l'homme traditionnel », peu perméable aux mutations sociales des rôles de genre, contre l'image du "nouvel homme ", par exemple. Qu'il s'agisse des hommes ou des femmes, toutes les personnes interrogées tendent à naturaliser la masculinité ; elles véhiculent ainsi ce que l'auteur appelle des "représentations constructionnistes "essentialisées" des rôles de genre » (p. 79).

\section{Des pratiques : restituer leur sens}

Au cœur du travail de Mendès-Leite se trouve la volonté de prendre systématiquement en compte le contexte et les significations localisées des pratiques sociales. Si le recours à une analyse compréhensive des pratiques n'est pas en soi inédit, l'auteur l'applique en revanche à des objets qui, jusqu'alors, avaient rarement été étudiés dans cette perspective. Ainsi en est-il de la pénétration anale dans l'article "Sens et contexte dans les recherches sur les (homo)sexualités et le sida : réflexion sur le sexe anal ». Le sens commun tend à faire du sexe anal l'un des vecteurs de l'assignation à une catégorie sociosexuelle : I'homosexualité. Pour ceux qui le pratiquent, il peut revêtir de multiples significations qui sont notamment fonction du rôle (pénétré ou pénétrant) et du comportement (passif ou actif) de chacun. La sodomie " passive » marque par exemple le "passage irréversible vers l'homosexualité pour les hommes français en quête d'une "protection" de leur identité hétérosexuelle " (p. 208). L'article " D'une norme à l'autre » montre d'ailleurs comment certains d'entre eux refusent de pratiquer le coït anal passif pour ne pas se penser et être perçus comme homosexuels. À ces stratégies de "refus", s'ajoutent des stratégies de " ruse » telles que le fait de négocier son identité de genre. Le texte met ainsi en avant la diversité des arrangements et des bricolages qui permettent de " vivre l'homosexualité sans la nommer » (p. 95).

Afin de restituer le sens des pratiques, Mendès-Leite accorde également une importance primordiale au travail de terrain. II a par exemple mené des observations sur les lieux de la sexualité " anonyme ", dont les résultats sont exposés dans l'article co-écrit avec Bruno Proth ("Civiliser la sexualité : des lieux de sexualité anonyme aux back-rooms »). Cette démarche empirique, qui n'est pas sans rappeler les travaux de Laud Humphreys (1970), permet ainsi de décrire avec précision deux contextes différents : les lieux extérieurs de drague (espaces publics) et les back-rooms (établissements commerciaux). À partir de leurs recherches ethnographiques, les auteurs font l'hypothèse que le développement d' " institutions " gays, tels les back-rooms et plus encore les saunas, constituerait une forme de " domestication de la sexualité masculine anonyme » (p. 110) et permettrait l'émergence d'une homosexualité « identitaire » (p. 135). Cette privatisation des lieux de drague favoriserait ainsi certaines formes, bien que limitées, de régulation des sexualités masculines, ainsi qu'un encadrement des pratiques " à risque ». 


\section{Des risques et des " protections imaginaires "}

Mendès-Leite relie la plupart de ses analyses à la question du risque face au VIH. Dans une perspective de prévention, il s'interroge sur les représentations du sida et les pratiques individuelles de gestion du risque. Il développe ainsi la notion de "protections imaginaires " (cf. son article de 1995, "Identité et altérité : protections imaginaires et symboliques face au sida ») qui marquera les recherches ultérieures sur les sexualités et le VIH. Selon lui, les individus adoptent des comportements préventifs qui relèvent d'un régime de rationalité différent de ceux préconisés par les épidémiologistes. Les discours officiels de prévention sont en fait réinterprétés en fonction des cadres cognitifs propres à chacun. Ces " arrangements » permettent aux individus de simplifier les contraintes qui en découlent et de négocier leur sexualité avec ces nouvelles normes. Ils procèdent ainsi «à une réappropriation des "consignes" de prévention, en leur redonnant du sens dans une autre perspective, même si à leurs yeux, le but préventif reste le même » (p. 218). Les hommes interrogés pensent par exemple réduire leur exposition au risque en sélectionnant avec attention leurs partenaires sexuels selon l'apparence, l'origine géographique, le style de vie, mais aussi le degré de familiarité (et donc de confiance) entretenu avec eux. Ils réinterprètent ainsi subjectivement les conseils des épidémiologistes en n'ayant pas de rapports sexuels avec une personne perçue comme contaminée.

De même, la "fidélité ", supposée limiter le risque de contamination en réduisant le nombre de partenaires, fait l'objet d'interprétations différentes. On voit, une fois encore, le travail effectué par Mendès-Leite pour déconstruire les mots et leurs réalités : les individus peuvent par exemple "faire la différence entre fidélité physique et fidélité émotionnelle » (p. 222) et se considérer "fidèle » sans opter pour l'exclusivité sexuelle ${ }^{4}$. Pour certains hommes bisexuels (cas abordé dans l'avant-dernière contribution), la réappropriation des discours sur la fidélité peut les inciter à ne pas recourir au préservatif lors de rapports sexuels avec des femmes, dans la mesure où ces dernières sont perçues comme plus stables et plus fidèles que les hommes. L'auteur met ainsi au jour un ensemble de mécanismes sociaux qui expliquent l'écart entre les préconisations de santé publique et les pratiques effectives des individus, alors placés dans un espace de protection imaginaire et symbolique.

Dans ses derniers travaux, Mendès-Leite s'intéresse également à l'expérience sociale des individus vivant avec le VIH (Banens, Mendès-Leite, 2006). Avec l'allongement de l'espérance de vie des personnes contaminées émerge une nouvelle problématique : comment vieillissent-elles avec le VIH ? Pour les femmes, évoquées dans le dernier article, l'expérience de la séropositivité sur le long terme induit trois façons de se représenter son propre corps : le " corps-douloureux », le " corps-monstrueux » et le " corps-vieilli » (p. 286). La démarche socio-anthropologique permet ainsi de repenser la question du VIH, de comprendre la diversité

${ }^{4}$ Sur ce sujet, voir également l'ouvrage récent de Marie-Carmen Garcia (2016) sur l'extraconjugalité durable. 
des comportements face au "risque » et enfin de saisir les trajectoires sociales et les représentations des personnes atteintes.

Tout au long de son œuvre, Mendès-Leite mène à la fois une réflexion sur les discours et les représentations des acteurs concernés, en s'appuyant également sur l'observation de leurs pratiques : autrement dit, l' " émicité " et la " descriptivité " chères à Jean-Pierre Olivier de Sardan (2004 : 44). Cette rigueur anthropologique, alliée à la finesse des analyses, contribue également à une réflexion sur les politiques de prévention ${ }^{5}$. En ce sens, il s'agit d'une recherche indéniablement marquée par le contexte de l'époque et l'engagement de l'universitaire dans les luttes militantes contre le sida.

Cet ouvrage posthume constitue ainsi une excellente synthèse de l'œuvre de Mendès-Leite et incite parallèlement à en approfondir la lecture. II rend également hommage au combat scientifique qu'il a mené : rendre visibles, puis légitimes, les sexualités comme objet de recherche.

\section{Bibliographie}

Banens M., Mendes-Leite R. (2006), Vivre avec le VIH, Paris, Calmann-Lévy.

Broqua C. (2011), "L'homosexualité comme construction sociale: sur le tournant constructionniste et ses prémices ", Genre, sexualité \& société, hors-série n 1, p. 2-17.

Mendes-Leite R., Deschamps C. (1997), « Des mots, des pratiques et des risques. La gestion différenciée de la parole et de la prévention du VIH chez des hommes à comportements bisexuels en France ", Sociologie et Sociétés, vol. 29, n 1, p. 99-111.

Foucault M. (1976), Histoire de la sexualité, t. I : La volonté de savoir, Paris, Gallimard.

GARCIA M.-C. (2016), Amours clandestines. Sociologie de l'extraconjugalité durable, Lyon, Presses universitaires de Lyon.

HumphreYs L. (1970), Tearoom Trade: Impersonal Sex in Public Places, New York, Aldine Publishing.

Olivier de SARDAN J.-P. (2004), "La rigueur du qualitatif. L'anthropologie comme science empirique ", Espaces Temps, n 84-86, p. 38-50.

WeEks J. (1977), Coming Out: Homosexual Politics in Britain from the Nineteenth Century to the Present, Londres, Quartet Books.

${ }^{5} C^{\prime}$ est d'ailleurs le sens des conclusions de la moitié environ des contributions reprises dans l'ouvrage que de rappeler cette utilité qui justifie également le financement de recherches en la matière. 